\title{
Discovering biomarkers for antidepressant response: protocol from the Canadian biomarker integration network in depression (CAN-BIND) and clinical characteristics of the first patient cohort
}

Raymond W. Lam¹, Roumen Milev², Susan Rotzinger ${ }^{3,4}$, Ana C. Andreazza ${ }^{4,5}$, Pierre Blier ${ }^{6}$, Colleen Brenner ${ }^{7}$, Zafiris J. Daskalakis ${ }^{4,5}$, Moyez Dharsee ${ }^{8}$, Jonathan Downar ${ }^{3,4}$, Kenneth R. Evans ${ }^{8,9}$, Faranak Farzan ${ }^{4,5}$, Jane A. Foster ${ }^{3,10}$, Benicio N. Frey ${ }^{10}$, Joseph Geraci ${ }^{3}$, Peter Giacobbe ${ }^{3,4}$, Harriet E. Feilotter ${ }^{8,9}$, Geoffrey B. Hall ${ }^{10}$, Kate L. Harkness ${ }^{11}$, Stefanie Hassel ${ }^{12}$, Zahinoor Ismail ${ }^{13}$, Francesco Leri ${ }^{14}$, Mario Liotti ${ }^{15}$, Glenda M. MacQueen ${ }^{13}$, Mary Pat McAndrews ${ }^{3}$, Luciano Minuzzi ${ }^{10}$, Daniel J. Müller ${ }^{4,5}$, Sagar V. Parikh ${ }^{16}$, Franca M. Placenza ${ }^{3}$, Lena C. Quilty ${ }^{4,5}$, Arun V. Ravindran ${ }^{4,5}$, Tim V. Salomons ${ }^{17}$, Claudio N. Soares ${ }^{18}$, Stephen C. Strother ${ }^{19}$, Gustavo Turecki ${ }^{20,21}$, Anthony L. Vaccarino ${ }^{8}$, Fidel Vila-Rodriguez ${ }^{1}$, Sidney H. Kennedy ${ }^{3,4,18^{*}}$ (D) and on behalf of the CAN-BIND Investigator Team

\begin{abstract}
Background: Major Depressive Disorder (MDD) is among the most prevalent and disabling medical conditions worldwide. Identification of clinical and biological markers ("biomarkers") of treatment response could personalize clinical decisions and lead to better outcomes. This paper describes the aims, design, and methods of a discovery study of biomarkers in antidepressant treatment response, conducted by the Canadian Biomarker Integration Network in Depression (CAN-BIND). The CAN-BIND research program investigates and identifies biomarkers that help to predict outcomes in patients with MDD treated with antidepressant medication. The primary objective of this initial study (known as CAN-BIND-1) is to identify individual and integrated neuroimaging, electrophysiological, molecular, and clinical predictors of response to sequential antidepressant monotherapy and adjunctive therapy in MDD.

(Continued on next page)
\end{abstract}

\footnotetext{
*Correspondence: sidney.kennedy@uhn.ca

Raymond W. Lam, Roumen Milev and Susan Rotzinger are Co-first authors.

${ }^{3}$ University Health Network, 399 Bathurst Street, Toronto, ON M5T 258,

Canada

${ }^{4}$ Department of Psychiatry, University of Toronto, 250 College Street, 8th

floor, Toronto, ON M5T 1R8, Canada

Full list of author information is available at the end of the article
} 
(Continued from previous page)

Methods: CAN-BIND-1 is a multisite initiative involving 6 academic health centres working collaboratively with other universities and research centres. In the 16-week protocol, patients with MDD are treated with a first-line antidepressant (escitalopram 10-20 mg/d) that, if clinically warranted after eight weeks, is augmented with an evidence-based, add-on medication (aripiprazole 2-10 mg/d). Comprehensive datasets are obtained using clinical rating scales; behavioural, dimensional, and functioning/quality of life measures; neurocognitive testing; genomic, genetic, and proteomic profiling from blood samples; combined structural and functional magnetic resonance imaging; and electroencephalography. De-identified data from all sites are aggregated within a secure neuroinformatics platform for data integration, management, storage, and analyses. Statistical analyses will include multivariate and machine-learning techniques to identify predictors, moderators, and mediators of treatment response.

Discussion: From June 2013 to February 2015, a cohort of 134 participants (85 outpatients with MDD and 49 healthy participants) has been evaluated at baseline. The clinical characteristics of this cohort are similar to other studies of MDD. Recruitment at all sites is ongoing to a target sample of 290 participants. CAN-BIND will identify biomarkers of treatment response in MDD through extensive clinical, molecular, and imaging assessments, in order to improve treatment practice and clinical outcomes. It will also create an innovative, robust platform and database for future research.

Trial registration: ClinicalTrials.gov identifier NCT01655706. Registered July 27, 2012.

\section{Background}

Depressive disorders, including Major Depressive Disorder (MDD), are highly prevalent and disabling conditions with substantial personal and societal costs [1]. MDD is now the second leading cause of disability worldwide [2] and contributes to excess mortality associated with many comorbid medical conditions [3]. Treatment of depressive disorders is based on empirical data and evidence-based guidelines, but treatment selection remains more of an art than a science. Hence, the discovery of clinical and biological markers, or biomarkers, of treatment response that would inform an individualized approach to depression treatment remains a research goal [4].

A major challenge to identify predictors (baseline characteristics that predict response), moderators (baseline characteristics that predict differential response to a specific treatment) and mediators (events or changes occurring during treatment that explains the response) is that MDD is a complex, heterogeneous condition. A variety of neurobiological and environmental influences, both independently and in combination with one another, can alter the clinical expression of MDD in terms of symptoms, severity, episode duration, response to treatment, and functional outcomes. As a result, no single intervention is effective for all people with depression. Current diagnostic systems such as the DSM-5 [5] can reliably codify depressive symptoms as criteria for MDD, but these symptoms are not unique to depression and, even if clustered together, may not represent a specific underlying disease process or a treatment substrate. Hence, the clinical entity termed "Major Depressive Disorder" represents only the final, external manifestations of an enormously complex, multi-level, multi-factorial process.
The Canadian Biomarker Integration Network in Depression (CAN-BIND) [6] was created with an aim to use an integrated approach to biomarker discovery. CAN-BIND draws on multidisciplinary expertise from investigative teams at 8 Canadian universities, all in active collaboration with the Ontario Brain Institute (OBI) [7] and Indoc Research (Toronto, ON, Canada). The Canadian Institutes of Health Research, academic institutions, and various industry partners provide additional funding and support (see Acknowledgements).

The overall goal of CAN-BIND is to identify predictors, moderators, and mediators of treatment response and non-response in people with MDD to guide clinical decision-making. CAN-BIND-1 uses an integrated clinical, neuroimaging and molecular approach with high-dimensional mathematical modeling techniques to specifically search for (a) baseline predictors and moderators of antidepressant and adjunctive agent response, (b) early treatment mediators of response (changes from baseline to 2 weeks), and (c) later treatment mediators of response (changes from baseline to 8 weeks, and to 16 weeks).

\section{Methods}

\section{Overview of protocol}

Patients with MDD are treated with open-label escitalopram $10-20 \mathrm{mg} / \mathrm{d}$ for 8 weeks. Responders $(\geq 50 \%$ reduction in Montgomery-Åsberg Depression Rating Scale [MADRS] score) continue on escitalopram for another 8 weeks, while non-responders have aripiprazole 2$10 \mathrm{mg} / \mathrm{d}$ added on to escitalopram for 8 weeks. Clinical, neuroimaging and molecular assessments are conducted at Baseline (Week 0) and Weeks 2 and 8; clinical and molecular assessments also are conducted at Weeks 4, 10, and 16; and additional brief clinical evaluations are completed at Weeks 6, 12, and 14. Clinical characterization 
assesses a broad palette of symptoms, functional outcomes, cognitive performance, personality dimensions, and recent and past life events.

\section{Participants}

Participants are recruited at 6 clinical centres: Vancouver (Djavad Mowafaghian Centre for Brain Health), Calgary (Hotchkiss Brain Institute), Toronto (2 sites: University Health Network and Centre for Addiction and Mental Health), Hamilton (St. Joseph's Healthcare Hamilton), and Kingston (Providence Care, Mental Health Services). Research Ethics Boards at each site approved the study. Recruitment draws upon outpatient-referral networks, community-based advertising, and dedicated knowledge translation (KT) activities.

Table 1 lists the inclusion and exclusion criteria for the patients with MDD. Healthy comparison participants

Table 1 Inclusion and exclusion criteria for patients

Inclusion Criteria
- Outpatients 18 to 60 years of age.
- DSM-IV-TR criteria for MDE in MDD, as confirmed by the MINI.
- Depressive episode duration $\geq 3$ months.
- Free of psychotropic medications for at least 5 half-lives (i.e. 1 week
for most antidepressants, 5 weeks for fluoxetine) before baseline.
- Score $\geq 24$ on the MADRS.
- Fluent in English, sufficient to complete the interviews and
self-report questionnaires.
Exclusion Criteria
- Diagnosis of Bipolar I or Bipolar II disorder.
- Any other psychiatric diagnosis that is considered the primary diagnosis.
- Any significant personality disorder diagnosis (e.g., borderline,
antisocial) that might interfere with participation in the protocol,
defined by clinician judgment.
- High suicidal risk, defined by clinician judgment.
- Substance dependence/abuse in the past 6 months.
- Significant neurological disorders, head trauma, or other unstable
medical conditions.
- Pregnant or breastfeeding.
- Psychosis in the current episode.
- High risk for hypomanic switch (i.e., history of antidepressant-
induced hypomania).
- Failed 4 or more adequatermined by the ATHF).
- Started psychological treatment within the past 3 months with the
- Contraindications to MRI.
asic interventions
-

DSM-IV-TR, Diagnostic and Statistical Manual of Mental Disorders, Fourth Edition, Text Revision; MDE, Major Depressive Episode; MDD, Major Depressive Disorder; MINI, Mini International Neuropsychiatric Interview; MADRS, Montgomery-Åsberg Depression Rating Scale; ATHF, Antidepressant Treatment History Form are 18-60 years of age, with no psychiatric or unstable medical diagnosis, and sufficient fluency in English to complete study procedures. They are matched to the patient group by sex and age distribution.

\section{Procedure}

At the Screening Visit, eligible participants provide written, informed consent for all study procedures. Each patient undergoes screening evaluations that include a full psychiatric consultation to confirm a diagnosis of MDD using the Mini International Neuropsychiatric Interview (MINI) [8]. Previous medication history (type, dose, and duration) is collected using the Antidepressant Treatment History Form (ATHF) [9]. A detailed medical history and listing of concomitant treatments, if any, are recorded. A reproductive/menstrual history is obtained from female patients. Patients undergo medical work-up that includes physical examination, height and body weight measurements, clinical laboratory assays, and 12lead electrocardiography (if indicated). Social background is collected using standardized reporting forms for demographic characteristics, handedness, ethnicity, education (expressed as years of formal schooling), marital status, occupational status, job classification, and household income. Participants are screened for contraindications to magnetic resonance imaging (MRI).

Figure 1 shows an overview of the protocol. At the Baseline Visit (Visit 1, Week 0), extensive clinical assessments are conducted, and blood and urine samples are obtained for molecular analysis. Participants also undergo the first of 3 sessions of structural and functional neuroimaging and electroencephalography (EEG), as described below. All patients start treatment with escitalopram and receive standardized clinical management based on the CANMAT clinical guidelines [10].

Neuroimaging/EEG, molecular and clinical assessments are conducted again at Weeks 2 and 8. Additional blood samples are collected at Week 4 for pharmacogenetic analysis, and at Weeks 2, 10, and 16 for medication levels. Blood chemistry screening, urinalysis, and body weight measurements are repeated at Week 16. At the conclusion of the study, patients are discharged into standard clinical care by a family physician and/or regular psychiatrist. Patients can also elect to enrol in a long-term naturalistic follow-up study that includes wellness monitoring using electronic mental health (e-Mental Health) tools.

Healthy comparison participants attend 5 study visits: Screening, Baseline, Week 2, Week 8, and Week 16. They complete the same assessments as patients but do not receive any treatment.

\section{Treatments}

In Phase 1, patients receive flexibly dosed escitalopram $(10-20 \mathrm{mg} / \mathrm{d})$ for 8 weeks. Patients are started at $10 \mathrm{mg} / \mathrm{d}$ 


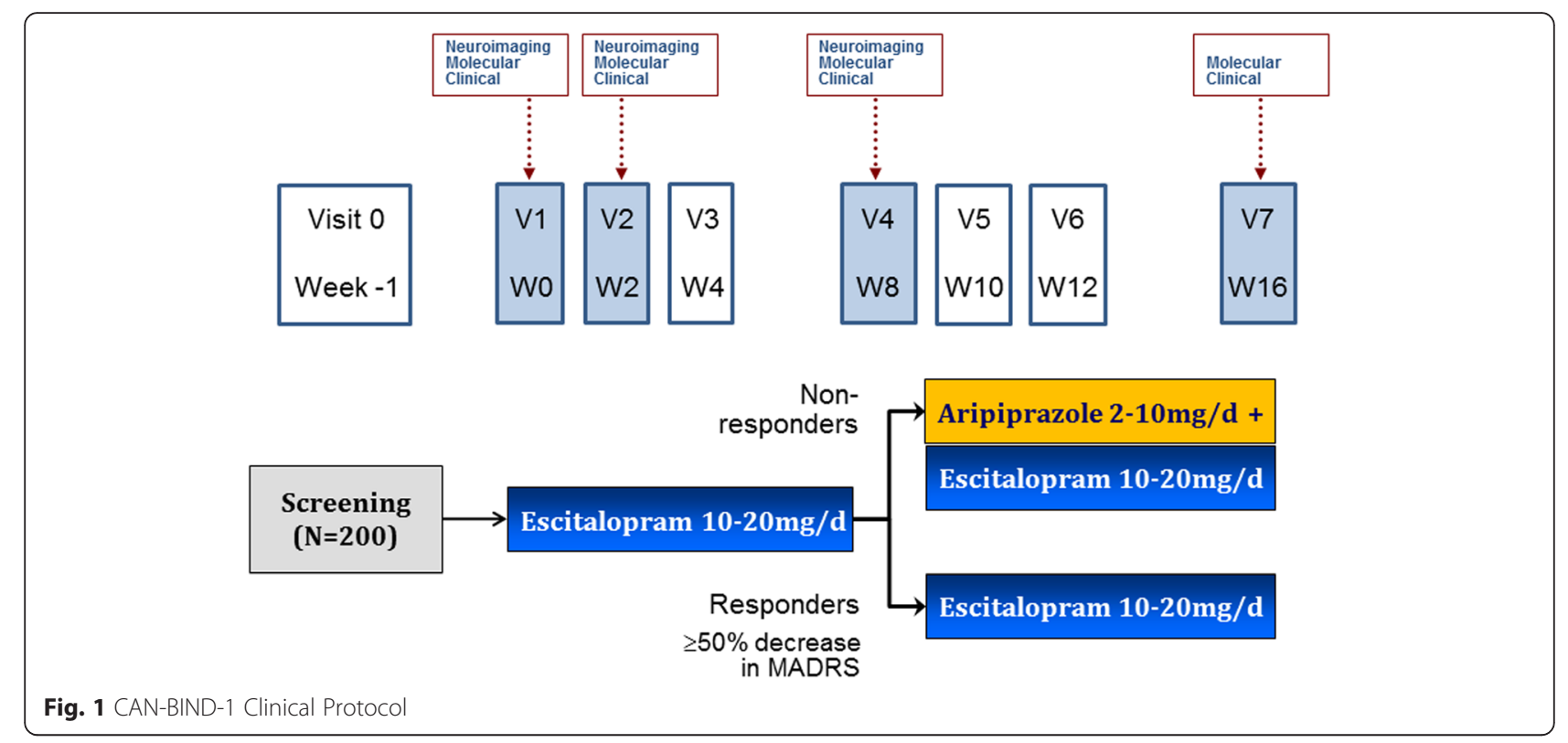

and increased to $20 \mathrm{mg} / \mathrm{d}$ at Week 2 if they do not achieve $\geq 20 \%$ reduction in MADRS score from baseline, and at Week 4 for those who do not achieve $\geq 50 \%$ reduction in MADRS.

In Phase 2, patients who achieve $\geq 50 \%$ reduction in baseline MADRS score at Week 8 are considered "responders" and continue on their effective dose of escitalopram for a further 8 weeks. Patients who do not achieve $\geq 50 \%$ reduction in MADRS are considered "non-responders" and receive flexibly dosed aripiprazole (2-10 $\mathrm{mg} / \mathrm{d})$, added to escitalopram, for a further 8 weeks. Dose increases of aripiprazole are recommended if patients do not achieve $\geq 50 \%$ reduction in MADRS after 2 or 4 weeks.

This standardized algorithm reflects usual clinical practice, is consistent with evidence-based treatment guidelines [11-13], and promotes consistency across all sites. Doses can be decreased at the discretion of the treating psychiatrist if patients do not tolerate higher doses. Treatment is open-label, and no randomization procedures are used. Medication adherence is monitored with pill counts at each visit.

Concomitant non-psychotropic medications for stable conditions are allowed at the discretion of the study psychiatrist, who can also permit use of vitamins, supplements, oral contraceptives, and non-prescription analgesics. Patients on pre-existing stable doses are allowed to continue on zopiclone up to $7.5 \mathrm{mg}$ prn or lorazepam 1-2 mg prn, to a maximum of 3 doses/week.

\section{Assessments}

\section{Clinical platform}

The clinical assessments (Table 2) are selected based on theoretical and clinical utility and to minimize respondent burden as much as possible. Raters received training, and inter-rater reliability was established using recorded interviews. Clinician-rated symptom and clinical measures include the Montgomery-Asberg Depression Rating Scale (MADRS), using the structured interview guide (SIGMA) to enhance reliability $[14,15]$, and Clinical Global Impression, Severity and Improvement scales (CGI-S and CGI-I) [16]. Depressive and other associated symptoms are explored in greater detail using the Depression Inventory Development (DID) semi-structured interview, as part of the International Society for CNS Drug Development's initiative to refine and validate a new measurement tool for use in clinical trials of MDD [17]. Patient-rated symptom scales include the Quick Inventory for Depressive Symptomatology (QIDS-SR) [18] and the Generalized Anxiety Disorder (GAD-7) scale [19]. Manic and hypomanic symptoms are assessed with the clinician-rated Young Mania Rating Scale (YMRS) [20] and the patient-rated Hypomania Check-List (HCL-32) [21].

Self-rated scales are used to assess functional impairment (Sheehan Disability Scale; SDS) [22] and occupational functioning (Lam Employment Absence and Productivity Scale; LEAPS) [23]. Quality of life is measured both generally (World Health Organization Quality of Life Assessment; WHOQoL-BREF) [24] and with a specific focus on depression (Quality of Life, Enjoyment and Satisfaction Questionnaire; Q-LES-Q) [25], using these 2 self-rated measures.

We also assessed other behavioural and dimensional constructs, including aversive and incentive motivation (Behavioural Inhibition System/Behavioural Activation System; BIS/BAS) [26], anhedonia (Dimensional Anhedonia Rating Scale; DARS; and Snaith-Hamilton Pleasure Scale; SHAPS) [27, 28], personality (NEO Five-Factor Inventory; NEO-FFI) [29] and pain (Brief Pain Inventory; BPI-SF) [30]. 
Table 2 Clinical characterization assessments

\begin{tabular}{|c|}
\hline Clinician-Administered Assessments \\
\hline Montgomery Asberg Depression Rating Scale (MADRS) \\
\hline Young Mania Rating Scale (YMRS) \\
\hline Clinical Global Impression (CGI) \\
\hline Depression Inventory Development (DID) Interview \\
\hline Toronto Side Effects Scale (TSES) \\
\hline Sexual Side Effects Questionnaire (SexFX) \\
\hline Childhood Experience of Care and Abuse (CECA) \\
\hline Life Events and Difficulties Schedule (LEDS) \\
\hline CNS Vital Signs (CNS-VS) computerized neuropsychological test battery \\
\hline National Adult Reading Test (NART) \\
\hline Self-Report Assessments \\
\hline Quick Inventory of Depressive Symptomatology, Self-Report (QIDS-SR) \\
\hline Generalized Anxiety Disorder 7-item scale (GAD-7) \\
\hline Hypomania Check-List (HCL-32) \\
\hline Brief Pain Inventory (BPI) \\
\hline Sheehan Disability Scale (SDS) \\
\hline Lam Employment Absence and Productivity Scale (LEAPS) \\
\hline Quality of Life, Enjoyment and Satisfaction Questionnaire (Q-LES-Q) \\
\hline World Health Organization Quality of Life Assessment (WHOQoL-BREF) \\
\hline Pittsburgh Sleep Quality Index (PSQI) \\
\hline Seasonal Pattern Assessment Questionnaire (SPAQ) \\
\hline Biological Rhythm Interview of Assessment in Neuropsychiatry (BRIAN) \\
\hline Dimensional Anhedonia Rating Scale (DARS) \\
\hline Snaith-Hamilton Pleasure Scale (SHAPS) \\
\hline Behavioural Inhibition/Behavioural Activation System (BIS/BAS) \\
\hline NEO Five-Factor Inventory (NEO-FFI) \\
\hline Experiences in Close Relationships (ECR-R) questionnaire \\
\hline List of Threatening Experiences (LTE) \\
\hline International Physical Activity Questionnaire (IPAQ) \\
\hline Brief Diet Questionnaire \\
\hline
\end{tabular}

Sleep, circadian rhythms, and seasonality are assessed using the Pittsburgh Sleep Quality Index (PSQI) [31], the Biological Rhythms Interview of Assessment in Neuropsychiatry (BRIAN) [32], and the Seasonal Pattern Assessment Questionnaire (SPAQ) [33]. Physical activity is measured using the self-rated International Physical Activity Questionnaire (IPAQ) [34], while dietary habits are assessed through a series of brief questions.

Assessment of environmental stressors includes adult patterns of attachment and recent stressful life events that are captured with self-report scales, including the Experience in Close Relationships (ECR-R) [35] scale, and the List of Threatening Experiences (LTE) [36], respectively.

Two clinical interviews are conducted by centralized, trained raters at Queen's University and facilitated by Medeo, a secure telehealth/videohealth service (Medeo, Vancouver, BC, Canada). The Childhood Experience of Care and Abuse (CECA) [37], administered at Week 4 to patients and at Week 2 to healthy participants, is a semi-structured interview that carefully assesses childhood maltreatment. The Life Events and Difficulties Schedule (LEDS) [38], administered at Week 16 to all participants, is a semi-structured contextual interview and rating system that assesses stressful life events that have occurred within 6 months of depression onset. Interviews are audiotaped and transcribed so that responses can be coded based on standard, manualized conventions.

CNS Vital Signs (CNS VS) is a computerized test battery used to assess memory, reaction time and psychomotor speed, complex attention, and cognitive flexibility [39]. The reliability of CNS VS is similar to that of conventional neuropsychological tests [40]. The CNS VS has a robust normative database and is sensitive to common causes of cognitive impairment. Analyses of cognitive data will be augmented with an estimate of premorbid intelligence obtained using the $\mathrm{Na}$ tional Adult Reading Test (NART) [41].

Medication side effects are documented with the clinician-rated Toronto Side Effects Scale (TSES) [42], which records the incidence, frequency, and severity of common adverse events, and the Sexual Functioning Questionnaire (SexFX), which evaluates specific sexual functioning [43]. Adverse Events are classified as mild, moderate or severe.

\section{Neuroimaging platform}

fMRI Structural and functional neuroimaging data are obtained on 3.0 Tesla (3 T) magnetic resonance imaging (MRI) systems using multicoll phased-array head coils. Among the 6 clinical sites, 4 different models of scanners are used, thus mandating an extensive and ongoing standardization and quality control process to ensure that data are comparable and usable [44]. The four models of scanners include Discovery MR750 3.0T (GE Healthcare, Little Chalfont, Buckinghamshire, UK), Signa HDxt 3.0T (GE Healthcare, Little Chalfont, Buckinghamshire, UK), MAGNETOM TrioTim (Siemens Healthcare, Erlangen, Germany), and Achieva 3.0T (Philips Healthcare, Best, Netherlands). Cross-site T1 piloting included a "human phantom", who travelled to each site for anatomical scans, and a manganese chloride $\left(\mathrm{MnCl}_{2}\right)$-based phantom model for progressive quantitative assessment of hydration based on signal intensity linearity characteristics [45]. Since the study's launch, each site has obtained monthly scans of 2 geometric phantoms (a spherical agar phantom developed by the Function Bioinformatics Research Network, and a custom-built cylindrical model using plastic LEGO blocks) $[46,47]$ to facilitate scanner calibration and troubleshooting over the long term. 
Following surveys and localization, the examination protocol includes a whole-brain T1-weighted turbo gradient echo sequence $(9 \mathrm{~min})$ at $1 \mathrm{~mm}^{3}$ resolution, repetition time $(\mathrm{TR})=6.2-1900 \mathrm{~ms}$, echo time $(\mathrm{TE})=2.7-3.5 \mathrm{~ms}$, flip angle $=8-15^{\circ}$, inversion time $(\mathrm{TI})=0-1100 \mathrm{~ms}$, field of view $(\mathrm{FOV})=220-256 \mathrm{~mm}$, matrix $256^{2}-512^{2}, 170-180$ contiguous slices at $1 \mathrm{~mm}$ thickness. A small Vitamin E capsule is placed as a stereotactic marker at the right temple to confirm subject orientation during image review [48]. Secondly, a whole-brain diffusion tensor imaging (DTI) series is obtained using 30 gradient directions at $2 \mathrm{~b}$-values (500 and $\left.1000 \mathrm{~s} / \mathrm{mm}^{2}\right)(5 \mathrm{~min})$ with an additional $3 \mathrm{im}$ ages at $\mathrm{b}=0 \mathrm{~s} / \mathrm{mm}^{2}$ for tensor construction at a final voxel resolution of $2.3 \times 2.3 \times 5 \mathrm{~mm}(1 \mathrm{~min})$. Functional MRI includes a 10-min resting state scan with eyes open using a fixation cross [49], obtained using a whole-brain $\mathrm{T} 2{ }^{*}$-sensitive blood oxygenation leveldependent (BOLD) echo planar imaging (EPI) series.

At the inception of the study, a BOLD EPI series was used during the Emotional Face Categorization/Conflict Task (2 runs of 7 min each) [50, 51]. After enrolling a cohort of 107 patients and 52 healthy participants, we substituted a reward paradigm and an implicit go no-go task. These tasks allow for the assessment of reward networks and attentional biases for affectively laden stimuli [52]. The substitution, rather than addition, of the functional tasks was necessary to maintain a feasible total time for participants in the scanner. The new tasks involve a 12-min hedonic function task [53, 54], where participants receive feedback and earn small monetary rewards while choosing correct responses among sets of visual stimuli and a 10-min affective go/no-go task to a series of stimuli that contain emotional content [55]. Stimulus sizes, instructions to participants, and support materials are standardized across sites. All behavioural data are captured using E-Prime software version 2.0 or higher (Psychology Software Tools, Sharpsburg, PA, USA).

Electroencephalography The fMRI tasks were translated into analogous versions for electroencephalography (EEG), which is carried out at 4 sites, again using several equipment models (Biosemi ActiveTwo [BioSemi, Amsterdam, Netherlands], BrainVision Recorder/QuickAmp [Brain Products, Munich, Germany], Compumedics NeuroScan [Compumedics USA, Charlotte, NC, USA], EGI Geodesic [Electrical Geodesics, Eugene, OR, USA]) that require cross-site consultation and standardization. All sites used a minimum of 64channel caps and conductive electrode gels, with 53 common electrodes identified across the different systems. EEGs are digitized continuously (bandpass $0.04-100 \mathrm{~Hz}$ with $1000 \mathrm{~Hz}$ sampling rate) and all electrodes are referenced to the vertex $(\mathrm{Cz})$ electrode.

At each session, participants complete a 10-min fixation resting state sequence with eyes open, followed by a 10-min resting state sequence with eyes closed. As with fMRI, the
Emotional Face Categorization/Conflict Task was used for EEG when the study began, but was later changed to a task querying anhedonia in MDD and an affective go/no-go task, also using E-Prime software. Ocular artifacts, such as blinks, saccades, and lateral movements, are removed by independent component analysis (ICA) as implemented in EEGLAB [56] and performed using MATLAB (MathWorks, Natick, MA, USA).

\section{Molecular platform}

Blood and urine samples for genomic and proteomic analyses are collected from all participants at Weeks 0, 2, 8 and 16 . Following blood draws at Weeks 0 , 2, and 8, total RNA is immediately isolated from leukocytes and stabilized using the LeukoLOCK filter apparatus (Thermo Fisher Scientific, Waltham, MA, USA), which also depletes globin mRNA to improve the utility of samples for expression profiling and other applications. For patients with MDD, additional blood samples are collected at Week 4 for pharmacogenetic analysis and at Weeks 2, 10, and 16 for drug levels.

Standard Operating Procedures (SOPs) for sample receipt and accessioning, nucleic acid extraction, quality assessment, data tracking, DNA and RNA preparation for assays, scanning, and feature extraction are in place.

Objectives of the molecular platform are to investigate 1) candidate biomarkers for disease state or drug response in baseline samples in patients and healthy participants, 2) global DNA alterations that may correlate with disease state or drug response using baseline samples, and 3) dynamic molecules in pre- and post- treatment samples to identify pathways of drug response and select additional targets for investigation by targeted methods.

De-identified specimens are transferred to and stored at the Douglas Mental Health University Institute biorepository in accordance with regulatory guidelines and best practices for biobanking. A subset of samples is transferred to other CAN-BIND sites for specific analyses. Material transfer agreements between sites were established at the outset of the study.

Targeted analyses include DNA single-nucleotide polymorphism (SNP) open-array analysis to identify and sequence variants that correlate with disease state or drug response [57], along with studies using established methods for profiling of mRNA and miRNA sequences, histone modifications, and methylation status across the genome [58]. Proteomic analysis by selected-reaction monitoring mass spectrometry (SRM-MS) with state-ofthe-art hybrid triple quadrupole/linear ion trap liquidchromatography mass spectrometry (5500 and 4000 QTRAPs), will be used for relative quantification of high-interest plasma proteins within biological pathways with purported relationships to MDD [59].

Exploratory analyses include RNA-Seq for miRNA from blood and from plasma using either a HiSeq (Illumina, 
San Diego, CA, USA) or Proton (Thermo Fisher Scientific, Waltham, MA, USA) platform. DNA oxidative damage to guanine will be evaluated by measuring levels of 8-hydroxy2-deoxyguanosine (8-OhdG) using a competitive ELISA analysis kit (StressMarq BioSciences, Victoria, BC, Canada). Cytosine oxidation will be measured using an ELISA-based assay to assess the levels of 5-hydroxymethylcytosine (MethylFlash ${ }^{\text {Tw }}$ Methylated DNA Quantification Kit; Epigentek Group, Farmingdale, NY, USA). Global DNA methylation (i.e., 5-methylcytosine) will be evaluated using an ELISA-based method (Sigma-Aldrich, Darmstadt, Germany). Inflammatory markers in blood will be measured using standard antibody-based immunoassays.

\section{Data management}

Each site has entered a standardized Participation Agreement with OBI to facilitate transfer of both raw and processed/de-identified data, in accordance with OBI's Governance Policy and with any specific conditions required by each institution's local legislative and/or ethical policies.

De-identified electronic data from all sites are aggregated for data analyses using multiple bioinformatics approaches. The OBI's Centre for Ontario Data Exploration ("BrainCODE", https://www.braincode.ca/) is an online neuroinformatics platform that allows researchers to collaborate across distances and work more efficiently, and ultimately, to promote new discoveries to improve patient care. BrainCODE provides the rare capability of supporting scientific inquiry and analytics across multiple brain diseases and modalities by integrating clinical, imaging, pathology, and genomics data. Standard variable definitions and formats are used so that investigators collect data consistently across studies and modalities. This will reduce variability in data collection and facilitate comparisons across diseases, merging of data sets and meta-analyses.

In storing sensitive patient information, Brain-CODE employs sophisticated security systems and utilizes Privacy by Design $(\mathrm{PbD})$ to protect privacy by embedding it into the design specifications of technologies, business practices, and physical infrastructure [7]. The OBI also works with several research ethics boards and related organizations to streamline the project review and approval process; and develops opportunities to link or integrate Brain-CODE with other provincial, national, or international databases to augment its analytical power. With this unique complement of capabilities, BrainCODE is the first database of its kind in the world.

Brain-CODE is deployed at the High Performance Computing Virtual Laboratory (HPCVL) data centre at Queen's University in Kingston, Ontario. The HPCVL is a Compute Canada high-performance computing consortium that supports regulatory-compliant (e.g., ICH E6, 21 CFR Part 11, HIPAA, PIPEDA) processes for securing privacy of healthcare data [60]. All data are collected, processed, maintained, and stored in Canada.

Online data/images can be accessed only on secure websites via restricted portals requiring unique usernames and passwords for each member of the study team. User profiles are assigned only to study personnel requiring access to enter/verify data, and credentials for each user are vetted by the program manager.

Specific data-collection platforms with Brain-CODE include:

1) Brain-CODE Subject Registry, a secure portal used by study centres in Ontario to link encrypted personal health information from study participants to provincial health/administrative databases;

2) OpenClinica Enterprise, a regulatory-compliant, web-based electronic data capture (EDC) system and database for demographic and historical data, diagnostic information, and clinician-rated assessments and scales (OpenClinica, Waltham, MA, USA);

3) LimeSurvey e-PRO Questionnaires, an open-source survey tool used by participants for direct entry of the 20 self-reported measures using a laptop or tablet while attending clinic visits (LimeSurvey, Hamburg, Germany);

4) SPReD (originally the Stroke Patient Recovery Research Database), a comprehensive online repository powered by the open-source Extensible Neuroimaging Archiving Toolkit (XNAT) imaging informatics platform [61]. Structural and functional MRI data are first converted to DICOM (Digital Imaging and Communications in Medicine) format at each site prior to uploading [62], whereas EEG data are uploaded as raw files for subsequent standardization to 58 channels and conversion into a universally readable format in EEGLAB. Supplementary results, such as behavioural and physiological data, and session notes, are uploaded through a special sub-process. All files must be correctly labeled in accordance with SPReD's organizational structure and naming conventions; an automated pipeline scans for errors on a daily basis; and

5) BASE (BioArray Software Environment) and LabKey, open-source laboratory-information management systems that enable tracking and management of proteomics and genomics workflows, experiments, and raw data [63, 64].

Clinical data support is provided by Indoc Research for OpenClinica and LimeSurvey, and by Rotman Research Institute/Baycrest Health Sciences for SPReD. For clinical and self-rated measures, source data verification is completed using a remote, risk-based monitoring system that includes centralized processes for cleaning and extracting data. 
Future CAN-BIND projects (see below) will use secure, customizable Research Electronic Data Capture (REDCap) tools for all clinical and self-reported data [65].

MRI data undergoes manual quality control (QC) procedures where trained raters check for visual artifacts and then grade scans as usable or rejected; in the latter case, rescanning is recommended if possible within the study timeline. For fMRI data, preprocessing includes conversion from DICOM to NIfTI (Neuroimaging Informatics Technology Initiative) format [66] prior to identifying the volume with the least motion, to which the remaining volumes are registered.

Converted EEG data are resampled to $512 \mathrm{~Hz}$ with channels re-referenced to $\mathrm{Cz}$ before digital filters are applied (high pass $=0.5 \mathrm{~Hz}$, low pass $=100 \mathrm{~Hz}$ ) and trigger codes are standardized.

For a period following study closure, CAN-BIND data are protected by OBI for the exclusive use of the investigative team and its collaborators. However, in the future, de-identified CAN-BIND data may be shared by OBI with other collaborators and third parties for research purposes. These datasets could be made available to other clinical research teams with similar datasets for comparison of treatment outcomes in other psychiatric conditions (such as dementia, schizophrenia, and bipolar disorder). Eligible third parties will be recognized researchers or organizations who have submitted detailed study plans and ethics boards approvals to the OBI. Before OBI discloses the de-identified data to any third party, it will enter into an agreement with the latter to protect confidentiality and ensure correct usage of data.

\section{Analyses}

We will examine several outcomes, including responders at Week 8 who maintained response at Week 16, responders at Week 8 who are non-responders at Week 16, non-responders at Week 8 who respond at Week 16 with augmentation, and non-responders at Weeks 8 and 16 , among other outcomes. A reasonably large dataset is required to study adequately the complex clinical, neuroimaging, and molecular factors that contribute to treatment outcomes. Our sample size target for this phase of the CAN-BIND program is 200 patients and 90 healthy participants (290 in total).

To identify standalone features that differ significantly between responders and non-responders, either at baseline or over the course of the study, parametric two-sample t-tests will be used for features with normally distributed data. Otherwise, the non-parametric Mann-Whitney $U$ test will be used. A significance threshold of $\alpha=0.05$ and multiple testing corrections will be required. Given an input list of up to 25,000 features, of which at least 250 are differentially expressed between responders and non-responders, assuming an overall false discovery rate of $5 \%$, and applying two- sample t-testing procedures, a minimum of 49 subjects per category are required for power of at least $90 \%$ to correctly identify a given feature which differs significantly between the categories. If nonparametric testing procedures are used, a minimum of 52 subjects per category are required to achieve the same power. Target recruitment for patients with MDD is 200. An estimated response rate of $60 \%$ would yield 120 responders and 80 non-responders. The planned sample size is sufficiently powered for both parametric and non-parametric univariate testing. Multivariate analysis and the development of prognostic signatures of escitalopram response will follow univariate analysis.

Data analyses will be conducted using a suite of commercial and open-source software tools installed on high-end workstations at HPCVL. While data from each analytical platform will be independently analyzed using the methods described above, important relationships (either correlational or causal) between modalities can be detected through an integration of data collected across assessment platforms for a given study subject.

To manage the integrated analyses of this complex data set, we have established a Data Science Advisory Team consisting of the Principal Investigators, domain-specific experts (e.g., molecular or imaging team members), informatics advisors, and operations support. This team will oversee processes for data cleaning, preprocessing, integration, quality control and overall analytics plans for each of the core research activities. Cross-domain as well as domain-specific data interrogation will be conducted by Working Groups responsible for applying specific analytic approaches according to their expertise and contributing to a master Statistical Analysis Plan. Conventional univariate and multivariate statistical approaches will address hypothesis-driven investigations, while a variety of analytics pipelines with both supervised and unsupervised techniques will allow exploratory analyses. Machine-learning approaches, including principle component analysis, random forests, and support vector machines will be used to identify subgroups of patients with shared symptoms and/ or biological features that could have clinical relevance.

\section{Progress to date}

Between 2013 and 2015, 159 participants (107 patients and 52 healthy participants) were screened and 134 ( 85 patients and 49 healthy participants) entered at baseline in the initial protocol with the Emotional Face Categorization/Conflict Task during MRI and EEG sessions, as noted above. Full neuroimaging data (to Week 8) were obtained from 71 patients and 45 healthy participants.

Table 3 lists the clinical characteristics of this first cohort $(N=134)$ that were evaluable at baseline. Independentsamples t-tests showed no significant demographic differences between patients and healthy participants. The patients were moderately depressed at baseline, as reflected 
Table 3 Clinical characteristics of the first cohort $(N=134)$

\begin{tabular}{|c|c|c|}
\hline Characteristic & $\begin{array}{l}\text { Patients with } \\
\text { MDD }(N=85)\end{array}$ & $\begin{array}{l}\text { Healthy participants } \\
(N=49)\end{array}$ \\
\hline Female:Male, N (\%) & 50:35 (59 \%:41 \%) & $32: 17$ (65 \%:35 \%) \\
\hline Age in years, mean (SD), range & $36.1(12.5), 19-61$ & 32.5 (10.2), 20-57 \\
\hline \multicolumn{3}{|l|}{ Ethnicity $^{a}, N(\%)$} \\
\hline Aboriginal & 0 & 0 \\
\hline Arab & $3(4 \%)$ & $1(2 \%)$ \\
\hline Asian & $9(11 \%)$ & $9(18 \%)$ \\
\hline Black & $1(1 \%)$ & 0 \\
\hline Latin American/Hispanic & $5(6 \%)$ & $2(4 \%)$ \\
\hline White & $59(69 \%)$ & $35(71 \%)$ \\
\hline Other & $5(6 \%)$ & $2(4 \%)$ \\
\hline Mixed & $3(4 \%)$ & 0 \\
\hline \multicolumn{3}{|l|}{ Marital status, N (\%) } \\
\hline Never Married & $48(57 \%)$ & $26(53 \%)$ \\
\hline Separated & $7(8 \%)$ & $1(2 \%)$ \\
\hline Married & $16(19 \%)$ & $12(25 \%)$ \\
\hline Divorced & $7(8 \%)$ & $3(6 \%)$ \\
\hline Domestic Partnership & $5(6 \%)$ & $6(12 \%)$ \\
\hline Widowed & $2(2 \%)$ & $1(2 \%)$ \\
\hline \multicolumn{3}{|l|}{ Occupational status, N (\%) } \\
\hline Working now & $45(53 \%)$ & $28(57 \%)$ \\
\hline $\begin{array}{l}\text { Disabled (permanent or } \\
\text { temporary) }\end{array}$ & $13(7 \%)$ & 0 \\
\hline Temporary leave & $5(3 \%)$ & 0 \\
\hline Looking, unemployed & $10(5 \%)$ & $3(6 \%)$ \\
\hline Student & $8(4 \%)$ & $15(31 \%)$ \\
\hline Retired & $1(1 \%)$ & 0 \\
\hline $\begin{array}{l}\text { If employed, number of hours } \\
\text { scheduled to work over the past } \\
2 \text { weeks: mean (SD) }\end{array}$ & $50.0(27.2)$ & $53.8(25.8)$ \\
\hline $\begin{array}{l}\text { If employed, number of hours } \\
\text { missed due to symptoms over } \\
\text { the past } 2 \text { weeks: mean (SD) }\end{array}$ & $10.5(18.2)$ & $0.1(0.6)$ \\
\hline Education, years: mean (SD) & $14.1(2.0)$ & $15.9(2.9)$ \\
\hline \multicolumn{3}{|l|}{ Age of onset of MDD, years: } \\
\hline mean $(S D)$, range & $20.6(10.7), 5-55$ & $\mathrm{n} / \mathrm{a}$ \\
\hline Single episode:Recurrent, N (\%) & 24:61 (29 \%:71 \%) & $\mathrm{n} / \mathrm{a}$ \\
\hline No. previous episodes, mean (SD) & $4.3(2.8)$ & $\mathrm{n} / \mathrm{a}$ \\
\hline \multicolumn{3}{|l|}{ Current episode duration } \\
\hline$\leq 12$ months & $39(46 \%)$ & $\mathrm{n} / \mathrm{a}$ \\
\hline $1-2$ years & $13(15 \%)$ & \\
\hline$>2$ years & $30(35 \%)$ & \\
\hline Unknown/not reported & $3(4 \%)$ & \\
\hline Median duration (range), months & $14.5(3-151)$ & \\
\hline \multicolumn{3}{|l|}{ Comorbidities $^{\mathrm{b}, \mathrm{c}}$} \\
\hline Substance-Related Disorders & $7(8 \%)$ & $\mathrm{n} / \mathrm{a}$ \\
\hline
\end{tabular}

Table 3 Clinical characteristics of the first cohort $(N=134)$ (Continued)

\begin{tabular}{|c|c|c|}
\hline Anxiety Disorders & \multicolumn{2}{|l|}{$67(79 \%)$} \\
\hline Eating Disorders & \multicolumn{2}{|l|}{$1(1 \%)$} \\
\hline Stable medical conditions & \multicolumn{2}{|l|}{$52(61 \%)$} \\
\hline \multicolumn{3}{|l|}{$\begin{array}{l}\text { Use of antidepressants during } \\
\text { current }\end{array}$} \\
\hline episode, $N(\%)$ & $60(71 \%)$ & $\mathrm{n} / \mathrm{a}$ \\
\hline $\begin{array}{l}\text { No. antidepressants used, } \\
\text { mean (SD) }\end{array}$ & $1.7(1.5)$ & \\
\hline Baseline MADRS, mean (SD) & $29.9(6.0)$ & $0.4(1.0)$ \\
\hline Baseline YMRS, mean (SD) & $2.2(1.8)$ & $0.3(0.7)$ \\
\hline Baseline CGI Severity, mean (SD) & $4.7(0.8)$ & $1.0(0.0)$ \\
\hline Baseline QIDS-SR, mean (SD) & $15.9(4.3)$ & $2.1(1.7)$ \\
\hline Baseline SDS, mean (SD) & $16.6(7.5)$ & $0.0(0.0)$ \\
\hline Baseline LEAPS, mean (SD) & $14.1(6.1)$ & $1.9(2.7)$ \\
\hline \multicolumn{3}{|c|}{$\begin{array}{l}\text { aCategories adapted from ethnic-origin groups listed in national census } \\
\text { questionnaires [67] } \\
{ }^{\mathrm{b}} \text { Based on DSM-IV-TR, as determined by the Mini International } \\
\text { Neuropsychiatric Interview } \\
\text { CPercentages may not add up to } 100 \% \text { because patients may have more than } \\
1 \text { comorbid condition } \\
\text { MDD Major depressive disorder, SD Standard deviation, MADRS Montgomery } \\
\text { Assberg Depression Rating Scale, YMRS Young Mania Rating Scale, CGI Clinical } \\
\text { Global Impression, QIDS-SR Quick Inventory of Depressive Symptomatology, } \\
\text { Self-Report, SDS Sheehan Disability Scale, LEAPS Lam Employment Absence } \\
\text { and Productivity Scale }\end{array}$} \\
\hline
\end{tabular}

by MADRS, QIDS-SR, and CGI-S mean scores, as well as moderately impaired as assessed by psychosocial (SDS) and work (LEAPS) functioning scales.

\section{Discussion}

The CAN-BIND study is a unique biomarker-discovery initiative in its mandate, scope, organizational structure, and integrative approach. First, the study collects systematic outcome data in the clinical, neuroimaging, and molecular domains in an agnostic fashion, with no preconceived identification of biomarkers for depression. Second, data are aggregated and integrated using a secure informatics platform that facilitates collaborative data sharing and rigorous quality-control processes across sites. Third, cross-domain and domain-specific analyses are undertaken using high-dimensional mathematical models supplemented with conventional statistical tools.

While CAN-BIND-1 focuses on pharmacotherapy, subsequent protocols will address biomarkers in treatment response using other evidence-based interventions for MDD, including transcranial magnetic stimulation, cognitive behaviour therapy, cognitive remediation, and others. Additional studies using CAN-BIND platforms to examine integrated biomarkers involve youth at risk for serious mental illness, suicide risk, and effects of childhood maltreatment on stress sensitivity and reward responsivity. CAN-BIND also operates a dedicated reverse-translation 
platform with various pre-clinical groups that investigate neuropharmacology of hedonic function in laboratory rats, zebrafish high-throughput screening, electrophysiological and behavioural impact of medications, and microRNA/inflammatory markers. CAN-BIND activities are broadly disseminated through multimodal, iterative knowledge translation/knowledge exchange initiatives that include ongoing collaboration with patients, family members, and other stakeholders in the community. Over time, CAN-BIND will expand with new collaborations with academic health centres, industry partners, and mental health networks.

\section{Conclusion}

CAN-BIND is a large Canadian collaborative research endeavor that is attempting to discover integrated clinical, imaging and molecular biomarkers of treatment response in MDD. It may also identify clinically relevant subtypes of depression and further our knowledge of the pathogenesis and pathophysiology of MDD. Given the multifaceted study design, we also expect to find novel psychobiological insights that will lead to the generation of new hypotheses to be validated in future studies.

\section{Ethics approval and consent to participate}

Research described in this article has been ethics approved at each participating clinical centre. The ethics committees include: University of British Columbia Clinical Research Ethics Board (Vancouver); University of Calgary Conjoint Health Research Ethics Board (Calgary); University Health Network Research Ethics Board (Toronto); Centre for Addiction and Mental Health Research Ethics Board (Toronto); Hamilton Integrated Research Ethics Board (Hamilton); Queen's University Health Sciences and Affiliated Teaching Hospitals Research Ethics Board (Kingston). Participants provided written, informed consent for all study procedures.

\section{Consent for publication}

Not applicable - this manuscript does not contain any individual persons' data.

\footnotetext{
Abbreviations

21 CFR Part 11: title 21 of the code of federal regulations [U.S.]; electronic records; electronic signatures, part 11; ATHF: antidepressant treatment history form; BASE: bioArray software environment; BIS/BAS: behavioural inhibition system/behavioural activation system; BOLD: blood oxygenation leveldependent; BPI-SF: brief pain inventory; Brain-CODE: Ontario brain institute centre for Ontario data exploration; BRIAN: biological rhythm interview of assessment in neuropsychiatry; CAN-BIND: Canadian biomarker integration network in depression; CANMAT: Canadian network for mood and anxiety treatments; CBT: cognitive-behavioural therapy; CECA: childhood experience of care and abuse; CGI: clinical global impression; CNS VS: central nervous system vital signs; Cz: vertex [in EEG]; DARS: dimensional anhedonia rating scale; DICOM: digital imaging and communications in medicine;

DID: depression inventory development; DNA: deoxyribonucleic acid; DSM5: diagnostic and statistical manual of mental disorders, fifth edition; DSM-IVTR: diagnostic and statistical manual of mental disorders, fourth edition, text
}

revision; DTI: diffusion-tensor imaging; ECR-R: experiences in close relationships; EEG: electroencephalography; ELISA: enzyme-linked immunosorbent assay; EPI: echo planar imaging; e-PRO: electronic version of patient-reported outcome; fMRI: functional magnetic resonance imaging; FOV: field of view; GAD: generalized anxiety disorder; GE: general electric; HCL-32: hypomania check-list, 32-item version; HIPAA: health insurance portability and accountability act [U.S.]; HPCVL: high performance computing virtual laboratory; Hz: hertz; ICH E6: international conference on harmonisation of technical requirements for registration of pharmaceuticals for human use, guidance on efficacy, 6 (good clinical practice); IPAQ: international physical activity questionnaire; iTBS: intermittent theta-burst stimulation; KT/KE: knowledge transfer/knowledge exchange; LEAPS: lam employment absence and productivity scale; LEDS: life events and difficulties schedule; LTE: list of threatening experiences; MADRS: montgomery-asberg depression rating scale; MDD: major depressive disorder; MDE: major depressive episode; MINI: mini international neuropsychiatric interview; miRNA: microRNA; mm: millimetres; MRI: magnetic resonance imaging; mRNA: messenger RNA; ms: milliseconds; NART: national adult reading test; NEO-FFI: NEO five-factor inventory; NIfTI: neuroimaging informatics technology initiative; OBI: Ontario brain institute; QC: quality control; QIDS-SR: quick inventory of depressive symptomatology, self-report; Q-LES-Q: quality of life, enjoyment and satisfaction questionnaire; QTRAP: quadruple/linear ion trap; PbD: privacy by design; PIPEDA: personal information protection and electronic documents act; PRN: pro re nata [i.e., in medicine, "as needed"]; PSQI: Pittsburgh sleep quality index; REDCap: research electronic data capture; RNA: ribonucleic acid; rTMS: repetitive transcranial magnetic stimulation; SDS: sheehan disability scale; SexFX: sexual side effects questionnaire; SHAPS: snaith-hamilton pleasure scale; SIGMA: structured interview guide for the MADRS; SMI: serious mental illness; SNP: single-nucleotide polymorphism; SOP: standard operating procedures; SPAQ: seasonal pattern assessment questionnaire; SPReD: stroke patient recovery research database; SRM-MS: selected-reaction monitoring mass spectrometry; TE: echo time [in MRI]; TR: repetition time [in MRI]; TSES: Toronto side effects scale; WHOQOL-BREF: world health organization quality of life assessment; XNAT: extensible neuroimaging archiving toolkit; YMRS: young mania rating scale.

\section{Competing interests}

RWL has received speaker and consultant honoraria or research funds from AstraZeneca, Brain Canada, Bristol-Myers Squibb, the Canadian Institutes of Health Research (CIHR), the Canadian Network for Mood and Anxiety Treatments, the Canadian Psychiatric Association, Eli Lilly, Janssen, Lundbeck, Lundbeck Institute, Medscape, Otsuka, Pfizer, Servier, St. Jude Medical, Takeda, the University Health Network Foundation, and Vancouver Coastal Health Research Institute. ACA has received funds form CIHR, OMHF, Ministry of Research and Innovation of Ontario, CAMH Foundation.

In the last 5 years, ZJD received research and equipment in-kind support for an investigator-initiated study through Brainsway Inc. ZJD has served on the advisory board for Sunovion, Hoffmann-La Roche Limited and Merck and received speaker support from Eli Lilly. He has also received support from the Ontario Mental Health Foundation (OMHF), the Canadian Institutes of Health Research (CIHR), the Brain and Behaviour Research Foundation and the Temerty Family and Grant Family and through the Centre for Addiction and Mental Health (CAMH) Foundation and the Campbell Institute. BNF has received grant/research support from Alternative Funding Plan Innovations Award, Brain and Behavior Research Foundation, Canadian Institutes of Health Research, Hamilton Health Sciences Foundation, J.P. Bickell Foundation, Ontario Brain Institute, Ontario Mental Health Foundation, Society for Women's Health Research, Teresa Cascioli Charitable Foundation, Eli Lilly and Pfizer, and has received consultant and/or speaker fees from AstraZeneca, Bristol-Myers Squibb, Canadian Psychiatric Association, CANMAT, Daiichi Sankyo, Lundbeck, Pfizer, Servier and Sunovion.

$\mathrm{ZI}$ has received research funding from CIHR, Canadian Consortium for Neurodegeneration and Aging, National Institute of Aging, Joan and Clifford Hatch Foundation, Katthy Taylor Chair in Vascular Dementia, Ontario AFP Innovation Fund and University of Calgary Department of Psychiatry, as well as consultant honoraria from BMS/Otsuka, Janssen, Lundbeck, Pfizer and Sunovion.

DJM has received research funds from CIHR, CFI, NARSAD, OMHF, NIH and the University of Toronto.

LM has received Grants/Research Support from Alternative Funding Plan Innovations Award, Brain \& Behavioral Foundation, Canadian Institutes of 
Health Research, Hamilton Health Sciences Foundation, Ontario Brain Institute, and Ontario Mental Health Foundation, and Speakers Bureau/ Honoraria from Bristol-Myers Squibb, Lundbeck, Canadian Psychiatric Association, and the Canadian Network for Mood and Anxiety Treatments. LCQ has received research funds from Ontario Mental Health Foundation, Gambling Research Exchange Ontario, Campbell Family Mental Health Research Institute, American Psychiatric Association, National Institutes of Health, and Canadian Consortium for Gambling Research.

AVR has received speaker and consultant honoraria or research funds from Bristol Myers Squibb, Canadian Depression Research and Intervention Network, Canadian Foundation for Innovation and the Ministry of Economic Development and Innovation, Canadian Institutes of Health Research, Grand Challenges Canada, Janssen, Lundbeck, Ontario Mental Health Foundation, Pfizer and Sunovion.

ALV has received consultant honoraria from Janssen and Roche. PB has received grant funding and/or honoraria for lectures and/or participation in advisory boards for Astra Zeneca, Bristol Myers Squibb, Eli Lilly, Forest, Euthymics, Janssen, Lundbeck, Merck, Otsuka, Pfizer, Pierre Fabre, Servier, Shire, Takeda, and Valeant.

FF has received funding from NARSAD and NSERC.

SVP has been a consultant to Takeda, Bristol Myers Squibb, Lundbeck; has had a research contract with Assurex; has equity in Mensante.

SCS is Chief Scientific Officer of ADM Diagnostics, LLC, Chicago. GT has an Investigator-initiated grant from Pfizer Canada, honorarium from Bristol-Meyers Squibb Canada and Janssen Canada.

PG has grants/research support from $\mathrm{CIHR}, \mathrm{NIH}$; and speaker's bureau/ honoraria from Bristol-Meyers-Squibb, Lundbeck, St.Jude Medical. JD has received research support from the Canadian Institutes of Health Research, Brain Canada, the National Institutes of Health, the Klarman Family Foundation, the Edgestone Foundation, and the Toronto General and Western Hospital Foundation, as well as travel stipends from Lundbeck and ANT Neuro, and in-kind equipment support for an investigator-initiated study from MagVenture.

FVR has received grant funding from Canadian Institutes of Health Research and Brain Canada

GMM has been on advisory board or speaker for Lundbeck, Lilly, Pfizer, Janssen RM has received speaker and consultant honoraria or research funds from AstraZeneca, Bristol-Myers Squibb, the Canadian Institutes of Health Research (ClHR), the Canadian Network for Mood and Anxiety Treatments, Eli Lilly, Janssen, Lundbeck, Ontario Mental Health Foundation, Otsuka, Paladin, Pfizer, Sunovion, and the University Health Network Foundation.

SHK has received funding from Allergan, Brain Canada, Bristol-Meyers Squibb, Canadian Institutes of Health Research, Janssen, Lundbeck, Lundbeck Institute, Medscape, Ontario Brain Institute, Otsuka, Pfizer, Servier, St. Jude Medical, Sunovion.

SR, HEF, GBH, KLH, ML, JAF, FL, JG, FMP, CB, MPM, KRE, SH, CNS, MD and TVS list no competing interests.

\section{Authors' contributions}

SHK, RWL, RM, SR, PB, KRE, BNF, PG, GMM, SVP, AVR, CNS, and GT made substantial contributions to overall study conception and design and to ongoing data collection and analyses. ACA, MD, KRE, JAF, JG, HEF, DJM, and GT contributed to development of the molecular platform and continue to contribute to data collection and/or analysis related to the platform. $\mathrm{GBH}$, SH, LM, TVM, SCS, JD, FVR contributed to the development of the fMRI platform and continue to contribute to data collection and/or analysis related to the platform. MPM, CB, FF, ML, JG contributed to the development of the EEG platform and continue to contribute to data analysis related to the platform. MD, KRE and ALV contributed to the development of the data management platform. SHK, RWL, RM, SR, ZJD, JD, KRE, JAF, BNF, PG, DJM, TVM, KLH, ZI, PB, FL, GMM, MPM, LM, SVP, FMP, LCQ, AVR, CNS, GT, ALV, and FVR contributed to the development of the clinical platform and continue to contribute to data collection and/or analysis related to the platform. RWL drafted the manuscript. All authors were involved in critical revisions of the manuscript. All authors read and approved the final manuscript.

\section{Acknowledgements}

CAN-BIND is an Integrated Discovery Program carried out in partnership with, and financial support from, the Ontario Brain Institute, an independent non-profit corporation, funded partially by the Ontario government. The opinions, results and conclusions are those of the authors and no endorsement by the Ontario Brain Institute is intended or should be inferred. Additional funding is provided by the Canadian Institutes of Health Research (CIHR), Lundbeck, Bristol-Myers Squibb, and Servier. Funding and/or in kind support is also provided by the investigators' universities and academic institutions. All study medications are independently purchased at wholesale market values. Members of the CAN-BIND Investigator Team are listed here: www.canbind.ca/ our-team/.

\section{Author details}

${ }^{1}$ University of British Columbia and Vancouver Coastal Health Authority, 2255 Wesbrook Mall, Vancouver, BC V6T 2A1, Canada. ${ }^{2}$ Queen's University, Providence Care, Mental Health Services 752 King Street West, Postal Bag 603, Kingston, ON K7L 7X3, Canada. ${ }^{3}$ University Health Network, 399 Bathurst Street, Toronto, ON M5T 2S8, Canada. ${ }^{4}$ Department of Psychiatry, University of Toronto, 250 College Street, 8th floor, Toronto, ON M5T 1R8, Canada. ${ }^{5}$ Centre for Addiction and Mental Health, 1001 Queen St. W, Toronto, ON M6J 1A8, Canada. 6 University of Ottawa Institute of Mental Health Research, 1145 Carling Avenue, Ottawa, ON K1Z 7K4, Canada. 'Loma Linda University, 24851 Circle Dr, Loma Linda, CA 92354, USA. ${ }^{8}$ Indoc Research, 258 Adelaide St. East, Suite 200, Toronto, ON M5A 1N1, Canada. ${ }^{9}$ Department of Pathology and Molecular Medicine, Queen's University, 88 Stuart Street, Kingston, ON K7L 3N6, Canada. ${ }^{10}$ McMaster University, and St. Joseph's Healthcare Hamilton, 1280 Main Street West, Hamilton, ON L8S4L8, Canada. ${ }^{11}$ Department of Psychology, Queen's University, Kingston, ON K7L 3N6, Canada. ${ }^{12}$ Aston University, Aston Triangle, Birmingham, West Midlands B4 7ET, UK. ${ }^{13}$ University of Calgary Hotchkiss Brain Institute, 2500 University Dr NW, Calgary, AB T2N 1N4, Canada. ${ }^{14}$ University of Guelph, 50 Stone Rd E, Guelph, ON N1G 2W1, Canada. ${ }^{15}$ Simon Fraser University, 8888 University Dr, Burnaby, BC V5A 1S6, Canada. ${ }^{16}$ Universisty of Michigan, 500 S State St, Ann Arbor, MI48109USA. ${ }^{17}$ University of Reading, Earley Gate, Whiteknights, Reading RG6 6AL, UK. ${ }^{18}$ St. Michael's Hospital, 193 Yonge St, Toronto, ON M5B 1M4, Canada. ${ }^{19}$ Rotman Research Institute at Baycrest Centre, 3560 Bathurst Street, Toronto, ON M6A 2E1, Canada. ${ }^{20}$ McGill University, 845 Rue Sherbrooke O, Montréal, QC H3A 0G4, Canada. ${ }^{21}$ Douglas Mental Health University Institute Frank B. Common (FBC) F-3145, 6875 LaSalle Boulevard, Montréal, QC H4H 1R3, Canada.

Received: 4 March 2016 Accepted: 18 March 2016

Published online: 16 April 2016

\section{References}

1. Ferrari AJ, Charlson FJ, Norman RE, Flaxman AD, Patten SB, Vos T, Whiteford $\mathrm{HA}$. The epidemiological modelling of major depressive disorder: application for the Global Burden of Disease Study 2010. PLoS One. 2013;8(7):e69637.

2. Global Burden of Disease Study 2013 Collaborators. Global, regional, and national incidence, prevalence, and years lived with disability for 301 acute and chronic diseases and injuries in 188 countries, 1990-2013: a systematic analysis for the Global Burden of Disease Study 2013. Lancet. 2015; 386(9995):743-800.

3. Walker ER, McGee RE, Druss BG. Mortality in mental disorders and global disease burden implications: a systematic review and meta-analysis. JAMA Psychiatry. 2015;72:334-41.

4. Collins PY, Patel V, Joestl SS, March D, Insel TR, Daar AS. Scientific Advisory Board and the Executive Committee of the Grand Challenges on Global Mental Health. Grand challenges in global mental health. Nature. 2011;475:27-30.

5. American Psychiatric Association. Diagnostic and Statistical Manual of Mental Disorders. 5th ed. Arlington: American Psychiatric Association; 2013.

6. Kennedy SH, Downar J, Evans KR, Feilotter H, Lam RW, MacQueen GM, Milev R, Parikh SV, Rotzinger S, Soares C. The Canadian Biomarker Integration Network in Depression (CAN-BIND): advances in response prediction. Curr Pharm Des. 2012;18(36):5976-89.

7. Stuss DT. The Ontario Brain Institute: completing the circle. Can J Neurol Sci. 2014;41(6):683-93.

8. Sheehan DV, Lecrubier Y, Sheehan KH, Amorim P, Janavs J, Weiller E, Hergueta T, Baker R, Dunbar GC. The Mini-International Neuropsychiatric Interview (M.I.N.I.): the development and validation of a structured diagnostic psychiatric interview for DSM-IV and ICD-10. J Clin Psychiatry. 1998;59(20):22-33. quiz 34-57.

9. Sackeim HA. The definition and meaning of treatment-resistant depression. J Clin Psychiatry. 2001;62(16):10-7.

10. Lam RW, Kennedy SH, Grigoriadis S, Mclntyre RS, Milev R, Ramasubbu R, Parikh SV, Patten SB, Ravindran AV. Canadian Network for Mood and Anxiety 
Treatments (CANMAT). Canadian Network for Mood and Anxiety Treatments (CANMAT) clinical guidelines for the management of major depressive disorder in adults. III. Pharmacotherapy J Affect Disord. 2009;117(1):S26-43.

11. Berman RM, Fava M, Thase ME, Trivedi MH, Swanink R, McQuade RD, Carson WH, Adson D, Taylor L, Hazel J, Marcus RN. Aripiprazole augmentation in major depressive disorder: a double-blind, placebo-controlled study in patients with inadequate response to antidepressants. CNS Spectr. 2009;14(4):197-206.

12. Berman RM, Marcus RN, Swanink R, McQuade RD, Carson WH, Corey-Lisle PK, Khan A. The efficacy and safety of aripiprazole as adjunctive therapy in major depressive disorder: a multicenter, randomized, double-blind, placebo-controlled study. J Clin Psychiatry. 2007;68(6):843-53.

13. Thase ME, Trivedi MH, Nelson JC, Fava M, Swanink R, Tran QV, Pikalov A, Yang $\mathrm{H}$, Carlson BX, Marcus RN, Berman RM. Examining the efficacy of adjunctive aripiprazole in major depressive disorder: a pooled analysis of 2 studies. Prim Care Companion J Clin Psychiatry. 2008;10(6):440-7.

14. Montgomery SA, Asberg M. A new depression scale designed to be sensitive to change. Br J Psychiatry. 1979;134:382-9.

15. Williams JB, Kobak KA. Development and reliability of a structured interview guide for the Montgomery Asberg Depression Rating Scale (SIGMA). Br J Psychiatry. 2008;192(1):52-8.

16. Guy W. ECDEU Assessment Manual for Psychopharmacology. Rockville: US Department of Health, Education, and Welfare Public Health Service Alcohol, Drug Abuse, and Mental Health Administration; 1976.

17. Kalali A, Vaccarino A, Evans K, Kennedy S, Kobak K, Sills T, Sheehan D, Williams J, Greist J, Schatzberg A. P.2.a.010 The depression inventory development workgroup: Results from the second validation study. Euro Neuropsychopharm. 2008;18(4):S298.

18. Rush AJ, Trivedi MH, Ibrahim HM, Carmody TJ, Arnow B, Klein DN, Markowitz JC, Ninan PT, Kornstein S, Manber R, Thase ME, Kocsis JH, Keller MB. The 16Item Quick Inventory of Depressive Symptomatology (QIDS), clinician rating (QIDS-C), and self-report (QIDS-SR): a psychometric evaluation in patients with chronic major depression. Biol Psychiatry. 2003;54(5):573-83.

19. Spitzer RL, Kroenke K, Williams JB, Löwe B. A brief measure for assessing generalized anxiety disorder: the GAD-7. Arch Intern Med. 2006;166(10):1092-7.

20. Young RC, Biggs JT, Ziegler VE, Meyer DA. A rating scale for mania: reliability, validity and sensitivity. Br J Psychiatry. 1978;133:429-35.

21. Angst J, Adolfsson R, Benazzi F, Gamma A, Hantouche E, Meyer TD, Skeppar P, Vieta E, Scott J. The HCL-32: towards a self-assessment tool for hypomanic symptoms in outpatients. J Affect Disord. 2005;88(2):217-33.

22. Leon AC, Olfson M, Portera L, Farber L, Sheehan DV. Assessing psychiatric impairment in primary care with the Sheehan Disability Scale. Int J Psychiatry Med. 1997;27(2):93-105.

23. Lam RW, Michalak EE, Yatham LN. A new clinical rating scale for work absence and productivity: validation in patients with major depressive disorder. BMC Psychiatry. 2009;9:78.

24. The WHOQOL Group. The development of the World Health Organization quality of life assessment instrument (the WHOQOL). In: Orley J, Kuyken W editors. Quality of Life Assessment: International Perspectives. Heidelberg: Springer Verlag; 1994. p. 41-57.

25. Endicott J, Nee J, Harrison W, Blumenthal R. Quality of Life Enjoyment and Satisfaction Questionnaire: a new measure. Psychopharmacol Bull. 1993. 29(2):321-6.

26. Carver CS, White TL. Behavioral inhibition, behavioral activation, and affective responses to impending reward and punishment: The BIS/BAS Scales. J Pers Soc Psychol. 1994;67(2):319-33.

27. Rizvi SJ, Quilty LC, Sproule BA, Cyriac A, Michael Bagby R, Kennedy SH. Development and validation of the Dimensional Anhedonia Rating Scale (DARS) in a community sample and individuals with major depression. Psychiatry Res. 2015;229(1-2):109-19.

28. Snaith RP, Hamilton M, Morley S, Humayan A, Hargreaves D, Trigwell P. A scale for the assessment of hedonic tone the Snaith-Hamilton Pleasure Scale. Br J Psychiatry. 1995;167(1):99-103.

29. Costa PT, McCrae RR. Revised NEO Personality Inventory (NEO PI-R) and NEO Five-Factor Inventory (NEO-FFI) Professional Manual. Odessa: Psychological Assessment Resources, Inc.; 1992.

30. Cleeland CS. Measurement of pain by subjective report. In: Chapman CR \& Loeser JD (eds.), Issues in Pain Measurement. Advances in Pain Research and Therapy, Volume.12. New York: Raven Press; 1989; 391-403.

31. Buysse DJ, Reynolds 3rd CF, Monk TH, Berman SR, Kupfer DJ. The Pittsburgh Sleep Quality Index: a new instrument for psychiatric practice and research. Psychiatry Res. 1989;28(2):193-213.
32. Giglio LM, Magalhães PV, Andreazza AC, Walz JC, Jakobson L, Rucci P, Rosa AR, Hidalgo MP, Vieta E, Kapczinski F. Development and use of a biological rhythm interview. J Affect Disord. 2009;118(1-3):161-5.

33. Magnusson A. Validation of the Seasonal Pattern Assessment Questionnaire (SPAQ). J Affect Disord. 1996;40(3):121-9.

34. Craig CL, Marshall AL, Sjöström M, Bauman AE, Booth ML, Ainsworth BE, Pratt M, Ekelund U, Yngve A, Sallis JF, Oja P. International physical activity questionnaire: 12-country reliability and validity. Med Sci Sports Exerc. 2003;35(8):1381-95.

35. Fraley RC, Waller NG, Brennan KA. An item response theory analysis of selfreport measures of adult attachment. J Pers Soc Psychol. 2000;78(2):350-65.

36. Brugha TS, Cragg D. The List of Threatening Experiences: the reliability and validity of a brief life events questionnaire. Acta Psychiatr Scand. 1990;82(1):77-81.

37. Bifulco A, Brown GW, Harris TO. Childhood Experience of Care and Abuse (CECA): a retrospective interview measure. J Child Psychol Psychiatry. 1994; 35(8):1419-35.

38. Bifulco A, Brown G, Edwards A, Harris T, Neilson E, Richards C, Robinson R. Life Events and Difficulties Schedule (LEDS-2): Vol. 1. Life events manual. London: Royal Holloway and Bedford New College, University of London; 1989.

39. Iverson GL, Brooks BL, Young AH. Identifying neurocognitive impairment in depression using computerized testing. Appl Neuropsychol. 2009;16(4):254-61.

40. Gualtieri CT, Johnson LG. Reliability and validity of a computerized neurocognitive test battery, CNS Vital Signs. Arch Clin Neuropsychol. 2006;21(7):623-43.

41. Nelson HE. National Adult Reading Test (NART): Test manual (Part1). Windsor: NFER-NELSON Publishing Company Ltd.; 1982.

42. Vanderkooy JD, Kennedy SH, Bagby RM. Antidepressant side effects in depression patients treated in a naturalistic setting: a study of bupropion, moclobemide, paroxetine, sertraline, and venlafaxine. Can J Psychiatry. 2002; 47(2):174-80.

43. Kennedy SH, Eisfeld BS, Dickens SE, Bacchiochi JR, Bagby RM. Antidepressantinduced sexual dysfunction during treatment with moclobemide, paroxetine, sertraline, and venlafaxine. J Clin Psychiatry. 2000;61(4):276-81.

44. Glover GH, Mueller BA, Turner JA, van Erp TG, Liu TT, Greve DN, Voyvodic JT, Rasmussen J, Brown GG, Keator DB, Calhoun VD, Lee HJ, Ford JM, Mathalon DH, Diaz M, O'Leary DS, Gadde S, Preda A, Lim KO, Wible CG, Stern HS, Belger A, McCarthy G, Ozyurt B, Potkin SG. Function biomedical informatics research network recommendations for prospective multicenter functional MRI studies. J Magn Reson Imaging. 2012;36(1):39-54.

45. Kurmis AP, Barber C, Slavotinek JP, Fazzalari NL. A MnCl2-based MR signal intensity linear response phantom. Radiol Technol. 2007;79(2):119-25.

46. Friedman $\mathrm{L}$, Glover $\mathrm{GH}$. Report on a multicenter $\mathrm{fMRI}$ quality assurance protocol. J Magn Reson Imaging. 2006;23(6):827-39.

47. Fonov VS, Janke A, Caramanos Z, Arnold DL, Narayanan S, Pike GB, Collins DL. Improved precision in the measurement of longitudinal global and regional volumetric changes via a novel MRI gradient distortion characterization and correction technique. In: Laio H, editor. Medical Imaging and Augmented Reality. Lecture Notes in Computer Science, vol. 6326. Berlin Heidelberg: Springer; 2010. p. 324-33.

48. Alzheimer's Disease Neuroimaging Insitiative MRI Technical Procedures Manual. [Internet]. 2013 [cited 2016 February 5]. Available from: http:// www.adni-info.org/Scientists/doc/ADNI2MRITrainingManualVersion5_ Oct2014_CLEAN.pdf.

49. Raichle ME, Snyder AZ. A default mode of brain function: a brief history of an evolving idea. Neuroimage. 2007;37(4):1083-90. discussion 1097-9.

50. Egner T, Etkin A, Gale S, Hirsch J. Dissociable neural systems resolve conflict from emotional versus nonemotional distracters. Cereb Cortex. 2008;18(6):1475-84.

51. Etkin A, Egner T, Peraza DM, Kandel ER, Hirsch J. Resolving emotional conflict: a role for the rostral anterior cingulate cortex in modulating activity in the amygdala. Neuron. 2006;51(6):871-82.

52. Elliott R, Rubinsztein JS, Sahakian BJ, Dolan RJ. The neural basis of moodcongruent processing biases in depression. Arch Gen Psychiatry. 2002;59(7): 97-604.

53. Hall GB, Milne AM, MacQueen GM. An fMRI study of reward circuitry in patients with minimal or extensive history of major depression. Eur Arch Psychiatry Clin Neurosci. 2014;264(3):187-98.

54. Vrieze E, Pizzagalli DA, Demyttenaere $K$, Hompes T, Sienaert P, de Boer $P$, Schmidt M, Claes S. Reduced reward learning predicts outcome in major depressive disorder. Biol Psychiatry. 2013;73(7):639-45.

55. Albert J, López-Martín S, Carretié L. Emotional context modulates response inhibition: neural and behavioral data. Neuroimage. 2010;49(1):914-21. 
56. Delorme A, Makeig S. EEGLAB: an open source toolbox for analysis of single-trial EEG dynamics. J Neurosci Methods. 2004;134:9-21.

57. Trevino V, Falciani F, Barrera-Saldaña HA. DNA microarrays: a powerful genomic tool for biomedical and clinical research. Mol Med. 2007; 13(9-10):527-41.

58. Einat P. Methodologies for high-throughput expression profiling of microRNAs. Methods Mol Biol. 2006;342:139-57.

59. Chiu CL, Randall S, Molloy MP. Recent progress in selected reaction monitoring MS-driven plasma protein biomarker analysis. Bioanalysis. 2009; 1(4):847-55.

60. High Performance Computing Virtual Lab [Internet]. Kingston, ON, Canada: High Performance Computing Virtual Lab; 2016 [accessed 2016 Jan 30]. Available from:https://www.hpcvl.org/security/security-policy

61. Gee T, Kenny S, Price CJ, Seghier ML, Small SL, Leff AP, Pacurar A, Strother SC. Data warehousing methods and processing infrastructure for brain recovery research. Archives Italiennes de Biologie. 2010; 148(3):207-17.

62. National Electrical Manufacturers Association. NEMA PS3 / ISO 12052, Digital Imaging and Communications in Medicine (DICOM) Standard. Virginia; 2016.

63. Vallon-Christersson J, Nordborg N, Svensson M, Häkkinen J. BASE $-2^{\text {nd }}$ generation software for microarray data management and analysis. BMC Bioinformatics. 2009;10:330

64. Nelson EK, Piehler B, Eckels J, Rauch A, Bellew M, Hussey P, Ramsay S, Nathe C, Lum K, Krouse K, Stearns D, Connolly B, Skillman T, Igra M. LabKey Server: an open source platform for scientific data integration, analysis and collaboration. BMC Bioinformatics. 2011;12:71.

65. Harris PA, Taylor T, Thielke R, Payne J, Gonzalez N, Conde JG. Research electronic data capture (REDCap) - A metadata-driven methodology and workflow process for providing translational research informatics support. J Biomed Inform. 2009;42(2):377-81.

66. Mark J. NIfTI-1 Data Format: Neuroimaging Informatics Technology Initiative [Internet]. 2007 [cited 2016 February 5]. Available from: http://nifti.nimh.nih. gov/nifti-1.

67. Statistics Canada; Ottawa, ON; Canada. Ethnic Origin Reference Guide, 2006 Census. Catalogue no. 97-562-GWE2006025. http://www12.statcan.gc.ca/ census-recensement/2006/ref/rp-guides/ethnic-ethnique-eng.cfm. Last modified: 4 May 2011.

\section{Submit your next manuscript to BioMed Central and we will help you at every step:}

- We accept pre-submission inquiries

- Our selector tool helps you to find the most relevant journal

- We provide round the clock customer support

- Convenient online submission

- Thorough peer review

- Inclusion in PubMed and all major indexing services

- Maximum visibility for your research

Submit your manuscript at www.biomedcentral.com/submit

) Biomed Central 